\title{
Explaining ecosystem multifunction with evolutionary models
}

\author{
Marc W. Cadotte,,${ }^{1,2,3,7}$ Stuart W. Livingstone, ${ }^{4}$ Simone-Louise E. Yasui,${ }^{1,2,5}$ Russell Dinnage, ${ }^{6}$ Jin-tian Li,${ }^{3}$ \\ Robin Marushia, ${ }^{1} \mathrm{~J}_{\mathrm{Ames}}$ Santangelo, ${ }^{2}$ and Wensheng Shu ${ }^{3}$ \\ ${ }^{1}$ Department of Biological Sciences, University of Toronto-Scarborough, 1265 Military Trail, Toronto, Ontario M1C 1 A4 Canada \\ ${ }^{2}$ Ecology and Evolutionary Biology, University of Toronto, 25 Willcocks Street, Toronto, Ontario M5S 3 B2 Canada \\ ${ }^{3}$ State Key Laboratory of Biocontrol, Key Laboratory of Biodiversity Dynamics and Conservation of Guangdong, College of Ecology \\ and Evolution, Sun Yat-sen University, Guangzhou 510275 China \\ ${ }^{4}$ Department of Physical and Environmental Science, University of Toronto-Scarborough, 1265 Military Trail, Toronto, \\ Ontario MIC 1 A4 Canada \\ ${ }^{5}$ School of Earth, Environmental and Biological Sciences, Queensland University of Technology (QUT), \\ Brisbane, Queensland 4001 Australia \\ ${ }^{6}$ Research School of Biology, Australian National University, 46 Sullivans Creek Road, Acton, \\ Australian Capital Territory 2601 Australia
}

\begin{abstract}
Ecosystem function is the outcome of species interactions, traits, and niche overlap - all of which are influenced by evolution. However, it is not well understood how the tempo and mode of niche evolution can influence ecosystem function. In evolutionary models where either species differences accumulate through random drift in a single trait or species differences accumulate through divergent selection among close relatives, we should expect that ecosystem function is strongly related to diversity. However, when strong selection causes species to converge on specific niches or when novel traits that directly affect function evolve in some clades but not others, the relationship between diversity and ecosystem function might not be very strong. We test these ideas using a field experiment that established plant mixtures with differing phylogenetic diversities and we measured ten different community functions. We show that some functions were strongly predicted by species richness and mean pairwise phylogenetic distance (MPD, a measure of phylogenetic diversity), including biomass production and the reduction of herbivore and pathogen damage in polyculture, while other functions had weaker (litter production and structural complexity) or nonsignificant relationships (e.g., flower production and arthropod abundance) with MPD and richness. However, these divergent results can be explained by different models of niche evolution. These results show that diversity-ecosystem function relationships are the product of evolution, but that the nature of how evolution influences ecosystem function is complex.
\end{abstract}

Key words: biodiversity; community ecology; ecosystem function; evolutionary models; multifunctionality; phylogenetic diversity; plant ecology.

\section{INTRODUCTION}

Healthy ecosystems provide a multitude of functions, with some delivering irreplaceable benefits to human societies (Costanza et al. 1997, Mace et al. 2012). Maximizing ecosystem multi-functioning means that carbon is sequestered (Chapin et al. 2000) - providing a potential pathway to help manage $\mathrm{CO}_{2}$ pollution; nutrients are cycled - helping to reduce excesses produced by modern industry (Spehn et al. 2005); pollinator food and habitat are provided (Ghazoul 2006) - contributing to increased crop yields (Klein et al. 2007); and storm water flow is regulated and air quality improved by pollution reduction - leading to higher quality of life in urban areas (Nowak et al. 2013, Hansen et al. 2015). Some of these functions have been shown to increase with species diversity

Manuscript received 25 January 2017; revised 6 July 2017; accepted 14 September 2017. Corresponding Editor: Lara Souza.

${ }^{7}$ E-mail: mcadotte@utsc.utoronto.ca
(Tilman et al. 2001, Balvanera et al. 2006, Cardinale et al. 2006), while others have not been adequately tested.

The nature of the effect of diversity on function depends on two main factors, the role of coexistence mechanisms and direct species contributions to these functions. Under coexistence, some functions are maximized when niche space is filled and more of the total available resource pool is utilized (Tilman 1999, Carroll et al. 2011, Turnbull et al. 2013). For example, community biomass increases with increasing richness if species utilize different resources (Gravel et al. 2011). Conversely, individual species contributions to function may be trait dependent, without a direct link to coexistence (Lavorel et al. 2011). For example, the abundances of two species using the exact same resources and with the same local fitness would be determined by neutral dynamics, but they would have unequal contributions to pollinator support if one was insect pollinated and the other pollinated by wind.

While these mechanisms are influenced by ecological processes, they are also the products of evolution, and can potentially be predicted by models of niche evolution. 
Niche differentiation allowing coexistence depends on a tendency for species to differ in meaningful ways - either through evolutionary drift in allopatry, which is often described by a Brownian motion model of ecological drift (e.g., Butler and King 2004, Cadotte and Davies 2016), or under divergent selection in sympatry (Clarke et al. 2015, Nuismer and Harmon 2015). Alternatively, species' traits can remain similar to ancestors because these trait values represent optimal states and are reinforced by stabilizing selection, as defined by Ornstein-Uhlenbeck models of evolution (Butler and King 2004). Traits that directly affect ecosystem function could have evolved under a number of evolutionary models, including one where key traits are tied into evolutionary diversification (Simpson 1944) and clades of close relatives possess these novel traits. For example, the evolution of novel flower types that increase attractiveness to pollinators or symbiotic relationships with soil bacteria that convert atmospheric nitrogen into ammonium are clade level traits that have not only influenced diversification but also directly contribute to ecosystem function.

Different evolutionary models make divergent predictions about diversity-function relationships. Several studies have tested the hypothesis that increased phylogenetic diversity (PD) should result in higher ecosystem function, and have compared the explanatory value of PD to that provided by species richness (Cadotte et al. 2008, Flynn et al. 2011, Cadotte 2013, 2015b, Venail et al. 2015), but all of these studies lack a specific expectation based on evolutionary models. We might naïvely assume that ecosystem function increases with both richness and mean pairwise phylogenetic distances (MPD) if niche differences are explained by the distances on phylogenetic trees (e.g., Cadotte 2015a), but this might not be what is precisely predicted by most evolutionary models. Under Brownian motion (BM), where niche differences are influenced by a single trait, we should expect that ecosystem function is explained by both richness and MPD, but that the variance in ecosystem function increases with increasing MPD, making it a weaker predictor than richness (Cadotte et al. 2017). There should be a weaker relationship between function and both richness and MPD under Ornstein-Uhlenbeck model if the selection strength keeping species at an optimal niche state is strong, reducing the evolution of complementary species. Speciation events that are associated with niche divergence will result in a positive relationship between phylogenetic distance and niche difference (Nuismer and Harmon 2015), and hence there should be a strong positive relationship between function and both richness and MPD, but we should expect that MPD should be a better predictor. Conversely, if single species or a group of closely related species provide maximal function then as diversity increases or more distantly related species added, the function might be reduced.

Although the evolutionary origins of single functions are central to the question of diversity-function relationships, ecosystems provide multiple functions simultaneously, and this multivariate nature of ecosystem function can be measured using statistical measures of 'multifunctionality' that combine individual functions into aggregate indices (Byrnes et al. 2014b). A number of recent papers have shown that multifunctionality measures that combine single functions generally increase with increasing diversity (Hector and Bagchi 2007, Gamfeldt et al. 2008, Zavaleta et al. 2010, Bradford et al. 2014, Lefcheck et al. 2015), and this positive relationship happens because either all single functions increase (Byrnes et al. 2014b) with diversity or diverse assemblages contain different species that provide or maximize different functions (Gamfeldt et al. 2008). By definition, these metrics obfuscate specific inferences about the ecological mechanisms influencing function, or the evolution underpinning these mechanisms (Byrnes et al. 2014a, Dooley et al. 2015), and so it is not entirely clear what an MPD-multifunction relationship should look like, but it should depend on the dominant evolutionary model explaining single functions.

We simulated species niche differences using three different models of niche evolution (Brownian motion, Ornstein-Uhlenbeck, and divergent selection) and a fourth model of the evolution of novel traits that directly influence ecosystem function regardless of coexistence via niche differences. We then examine the relationships between 10 measured ecosystem functions and both richness and mean pairwise phylogenetic distance from a field experiment and infer how evolution has shaped ecosystem function. Finally, we examine how multifunctionality indices capture the fact that different models of evolution influence individual functions.

\section{Materials ANd Methods}

\section{Simulations}

We created simulations using the $\mathrm{R}$ programming language (R Development Core Team 2013) to examine how the four different models of evolution (Brownian motion (BM), niche divergence (ND), Ornstein-Uhlenbeck (OU), and evolution of novel traits (NT)) predict ecosystem function (see supporting information for $\mathrm{R}$ code). In all cases described herein, simulations were run 1,000 times, with random 64-species phylogenetic trees created in each iteration, assemblages contain 1 to 4 species (to match the experimental design described in the next section), and for the $\mathrm{BM}, \mathrm{OU}$, and ND, the niche is a single resource axis bounded from 0 to 1 , with species' niches being normally distributed about an optimal niche value that evolves according to the specific evolutionary model, and the final niche curve for each species has an average standard deviation of 0.2. The ultrametric phylogenetic trees used in these simulations were created using the rcoal function in the ape package (Paradis et al. 2004).

Brownian motion (BM) niche evolution.-We evolved species' optimal niche values across the random phylogenetic tree with the ancestral niche value set at 0 using 
the rTraitCont function in the R package APE (Paradis et al. 2004). The rate of evolution (sigma) was held constant at 1 . We then rescaled the 64 optimal niche values to be between 0 and 1 and added on the normally distributed niche centred at the niche values by drawing minimum and maximum values from a normal distribution. A species niche is a certain proportion of the niche axis occupied by this niche distribution. Niche distributions that extended beyond 0 or 1 were truncated.

Ornstein-Uhlenbeck (OU) evolution.-We evolved species differences following an OU model using a similar set of procedures as described for BM evolution. The rate of evolution (sigma) was again set to 1 , and the strength of selection (alpha) was set to 10 .

Niche divergent evolution (ND).-Whereas BM produces a triangular relationship between phylogenetic distance and niche difference (Cadotte et al. 2017), where close relatives are very similar and distantly related species show high variance in similarity, divergent niche evolution creates a linear relationship between phylogenetic distance b) ND: Divergent niche
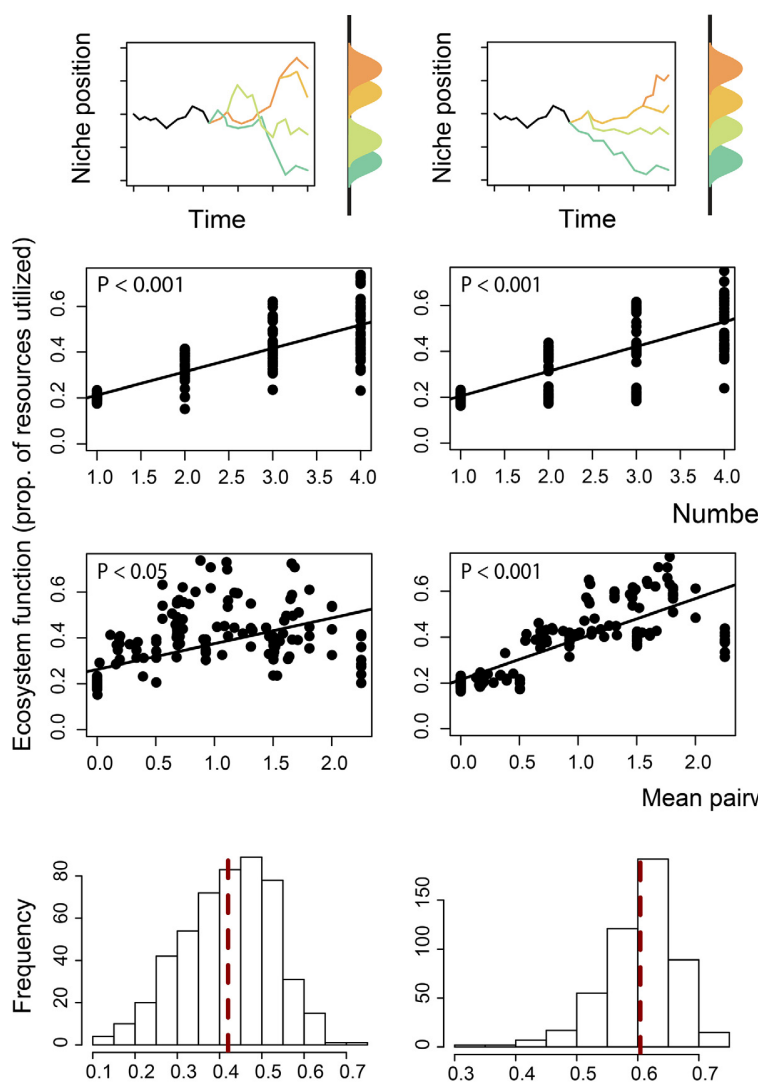

and ecological differences (Nuismer and Harmon 2015). To recover this linear relationship we selected a species in the center of the phylogeny and used the patristic distances from this species to all others to scale niche differences multiplied by a normally distributed error term to introduce some "noise" into the relationship between phylogenetic distance and niche difference. Once again niches were normally distributed around this optimal niche value (see description in the previous section) and truncated if less than 0 or greater than 1 .

Evolution of novel traits (NT).- Here we wanted to model the evolution of traits that contribute to ecosystem function directly rather than via niche differences. We evolved this effect trait directly using a BM model. We then identified all clades in the 64-species phylogeny with between four and eight species and randomly selected one of these clades and multiplied the evolved trait values by a constant ( $\mathrm{x} 3$ in our simulations). These traits were then scaled by the maximum trait value to represent species relative contributions to ecosystem function (see cartoon at the top of Fig. 1).

c) OU: Ornstein-Uhlenbeck

d) NT: Evolution of novel traits
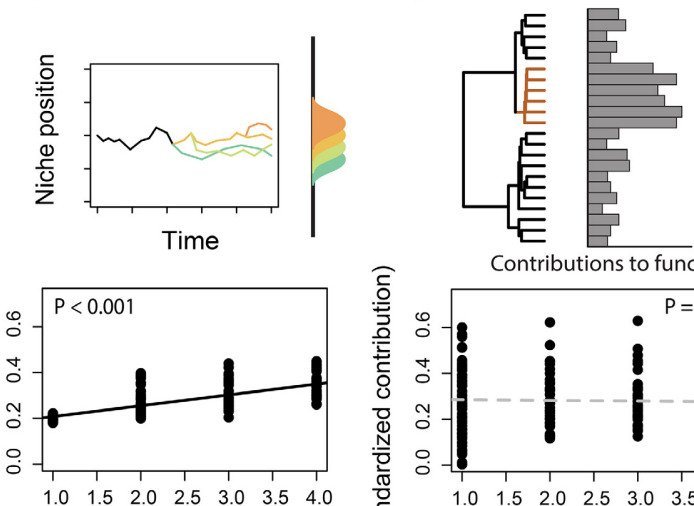

Number of species
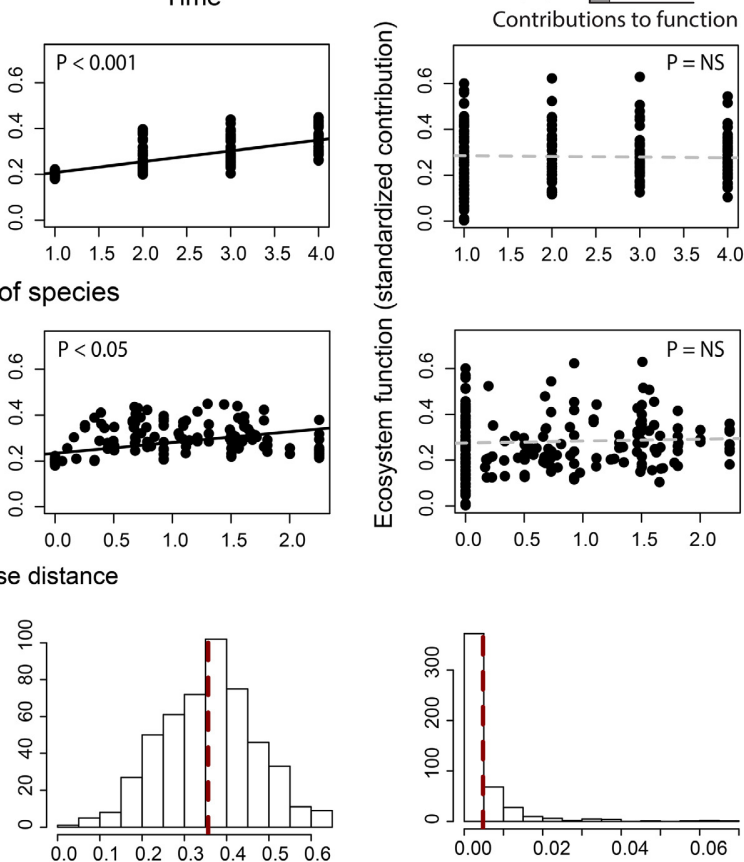

FIG. 1. The simulated relationships between both species richness and phylogenetic diversity with ecosystem function under three different evolutionary mechanisms: (a) Brownian motion evolution; (b) Ornstein-Uhlenbeck model; (c) divergent niche evolution; and (d) the evolution of novel traits. The relative explanatory power of the function-MPD relationships are shown with the distribution of R-squared values (bottom row). 
Turning niches and traits into ecosystem function.-The above four routines produced niche or trait values that we then used in simulations of ecosystem function. For the niches produced by $\mathrm{BM}, \mathrm{OU}$, and $\mathrm{ND}$, we created monoculture values as the proportion of the niche axis occupied by the niche distributions of species. For polycultures, we randomly sampled two, three, or four species and calculated the proportion of the niche axis occupied by the species, while not double counting the overlapping portions of the niche distributions. In these simulations, ecosystem function is simply the proportion of the niche axis being utilized by an assemblage and assumes that all species coexist (e.g., there are minimal fitness differences, which are much less than niche differences). This concept of ecosystem function matches those functions that depend on the amount of the available resources being utilized, biomass production for example (Tilman 1999).

For the direct trait contributions to ecosystem function from the NT model, we took the standardized trait values described above as monoculture values. For polycultures, we assumed that this contribution was diluted by other species by dividing this contribution by the number of species in polyculture, and calculated ecosystem function as the direct trait contribution multiplied by species proportional abundances, which were simply $1 / \mathrm{S}$, where $\mathrm{S}$ is the number of species in polyculture.

For each polyculture in the above simulations, we also recorded the number of species and the mean pairwise phylogenetic distance (MPD).

Analysis of simulation results. - For individual functions produced by these four evolutionary models, we used linear models using either the number of species or MPD to predict ecosystem function. In order to assess which evolutionary model produced the strongest relationships between ecosystem function and MPD, we ran simulations 1,000 times and examined the distribution of $\mathrm{R}$-squared values from linear models.

We also ran simulations and calculated multifunctionality by standardizing each individual function (Byrnes et al. 2014b) and regressing the average function with both species number and MPD. We simulated eight ecosystem functions, two from each of the above three evolutionary models and compared this to eight ecosystem functions produced by BM alone to determine how mixing ecosystem functions from different models influences multifunction measures.

\section{Field experiment}

This experimental design is explained in detail in Cadotte (2013), but briefly the experiment was conducted in an old field at the University of Toronto's Koffler Scientific Reserve $\left(44^{\circ} 02^{\prime}\right.$ N, $79^{\circ} 31^{\prime}$ W). In 2010, One hundred $2 \times 2 \mathrm{~m}$ plots were evenly spaced, separated by $1 \mathrm{~m}$ and seeded with 1, 2 or 4 species from a pool of 17, including: Andropogon gerardii (Vitman, Poaceae); Schizachyrium scoparium ([Michx.] Nash, Poaceae); Elymus canadensis (L., Poaceae); E. trachycaulus ([Link) Gould ex Shinners, Poaceae); Asclepias tuberosa (L., Asclepiadaceae); Doellingeria umbellata ([Mill.] Nees, Asteraceae); Liatris cylindracea (Michx., Asteraceae); Rudbeckia hirta (L. Asteraceae); Solidago nemoralis (Aiton, Asteraceae); Oligoneuron album ([Nutt.] G. L. Nesom, Asteraceae); Desmodium canadense (L., Fabaceae); Lespedeza capitata (Michx., Fabaceae); Monarda fistulosa (L., Lamiaceae); Pycnanthemum tenuifolium (Schrad., Lamiaceae); P. virginianum ([L.] T. Dur \& B. D. Jacks. Ex B. L. Rob \& Fernald, Lamiaceae); Penstemon digitalis (Nutt. Ex Sims, Scrophulariaceae); and P. hirsutus (L. Willd., Scrophulariaceae).

As described in Cadotte (2013), four species did not germinate (D. umbellata, L. cylindracea, O. album and P. digitalis). In late fall 2010, L. cylindricea and $O$. album were replaced with Solidago altissima (L., Asteraceae), and D. umbellata and P. digitalis were dropped from the experiment and several of the 4-species plots became 3 -species treatments, and several of the 2 -species plots became monocultures.

The polycultures were crossed with three treatments: (1) short; (2) medium; and (3) long phylogenetic distances. For the medium treatment, plots contain moderately distantly related species, or a mix of close and distant relatives. All species monocultures were replicated three times, small and large phylogenetic distance treatments were replicated 7 times each for each richness level, and the medium treatments were replicated 9 times, for each richness level. Plots we weeded throughout the growing season to exclude unplanted species.

Sampling functions.-We sampled 10 different community-level functions that span a number of different influences of plant diversity including resource dynamics, energy flow, and support for other trophic levels.

Soil nitrogen.-Composite soil samples were collected to a depth of $20 \mathrm{~cm}$ from each plot in June and August of 2013. Samples were air-dried, sieved, and analyzed for nitrogen with dry combustion (LECO FP428). Delta N values represent the change in percent soil nitrogen from June to August. Here, a decrease in Delta N across the growing season indicates a positive functional response, and vice versa (following Scherer-Lorenzen et al. 2003).

Total arthropod richness and abundance.-Each plot was sampled twice (late July and mid August 2012) using sweep nets. The nets were rapidly passed through the vegetation in each plot 30 times and insects were preserved in ethanol. Species were identified to genus or species using Marshall (2006) and counted.

Biomass production and litter-In mid August 2012, 2013, and 2014, $0.1 \times 1 \mathrm{~m}$ strips were delineated in each 
plot, not overlapping with previous years samples and at least $0.25 \mathrm{~m}$ away from any plot edge. All aboveground biomass was removed by cutting vegetation at soil surface, and was sorted into constituent species as well as litter (i.e., dead or dying material not connected to a living stem). All samples were dried in a VWR drying oven (VWR International, Radnor, PA, USA) at $50^{\circ} \mathrm{C}$ for $2 \mathrm{~d}$, and then weighed using a Mettler Toledo ML Series precision balance (Mettler Toledo Canada, Mississauga, $\mathrm{ON}$, Canada). We averaged the biomass estimates across all $3 \mathrm{yr}$ in our analysis.

Damage reduction. - In August 2014, ten plants for each species were randomly selected in each plot. For each sampled plant, we counted the total number of leaves, the number of leaves exhibiting insect herbivore damage, the number of leaves exhibiting disease damage, and estimated the percent leaf area damaged. For our analyses we calculated the proportion of damaged leaves (by both herbivores and disease) and estimated the reduction in damage $(\phi)$ in plot $i$ as the inverse of the log response ratio:

$$
\phi_{i}=-\sum_{j}^{S_{i}} \log \left(\frac{d_{j i}}{\bar{d}_{j}}\right)
$$

where $S_{i}$ is the number of species in polyculture $i, d_{j i}$ is the proportion of leaves diseased or damaged for species $j$ in plot $i$, and $\bar{d}_{j}$ is the average monoculture proportion of leaves diseased or damaged for species $j$.

Decomposition.-In late June 2013, we placed two nylon mesh bags filled with dried and weighed Solidago altissima leaves in each plot. Bags were collected after 1 and 2 months and the contents were dried and weighed. Here we show the mass lost after 2 months as our measure of decomposition. We chose $S$. altissima as our indicator species as it was the most abundant species surrounding our experimental plots, but we did not assess within species diversity which could influence decomposition and other functions (Crutsinger et al. 2006, Wang et al. 2014).

Flower production.-From the experimental species pool, 10 of the plant species produced flowers; however, only 9 flowered during the 2013 field season. Weekly counts of flower abundance for those 9 species were taken from early June to the end of September 2013. Plots with large abundances of flowers were quartered and the total number of flowers was estimated by multiplying the number of flowers counted in the randomly selected quarter by 4 . The flower abundances for the Solidago species were estimated based on the number of flowering branches found on a single plant, these estimates were in reference to fully counted Solidago plants. Missed flower counts for some observation days were estimated using mean interpolation.
Pollinator abundance.-Pollinator abundance was measured during the 2013 field season using biweekly 10-min plot observations. Based on personal observations and because rare flowers are more stochastic, plots with less than 10 flowers were not observed as we were unlikely to observe visits. During the 10-min sampling period any insect that landed on a flower within the plot, including accidental visitors, were counted as a potential pollinator. Plot observations occurred between 10 am to $3 \mathrm{pm}$, and were only conducted when sunny or partially cloudy. We recorded pollinators to morpho-species, and the number of times that type of pollinator was observed.

Structural complexity.-Structural complexity refers to the three-dimensional habitat structure provided by the vegetation assemblage, which represents habitat heterogeneity for other tropic levels (Beals 2006). Here we use a simplifying definition of the structural complexity of an assemblage as the number of leaf layers between the ground and top of the plant canopy (e.g., leaf area index - LAI). We measured LAI using the LP-80 AccuPAR PAR/LAI ceptometer (Decagon Devices, Inc. Pullman, WA, USA), which estimates LAI by the fraction of photosynthetically active radiation (PAR) standardized by the average leaf absorption of light.

Phylogeny. - The phylogeny construction is described in Cadotte (2013), but briefly, GenBank (Benson et al. 2005) was queried for five commonly sequenced genes: matK, rbcl, ITS1, ITS2 and 5.8s, for each species plus two representatives of early diverging angiosperm lineages as outgroup species, Amborella trichopoda (Baill.) and Magnolia grandiflora (L.). Sequences were aligned using MUSCLE (Edgar 2004) and models of nucleotide substitution for each gene were selected using the Akaike Information Criterion, as implemented in Modeltest (Posada and Crandall 1998, 2001).

The maximum likelihood phylogeny using the PHYML algorithm (Guindon and Gascuel 2003, Anisimova and Gascuel 2006) was used to estimate the phylogeny. The tree was made ultrametric using a semiparametric rate smoothing method (Sanderson 2002) to transform the phylogeny using the chronopl function in the R package ape (Paradis et al. 2004) and the tree was time calibrated to 160 million yr.

Analyses.-We used general linear models to estimate the ability of mean pairwise phylogenetic distance to explain variation in ecosystem function for each of the ten functions. We also calculated multifunctionality by standardizing each individual function (Byrnes et al. 2014b) and regressing the average function with MPD. We also examined threshold approaches advocated by (Byrnes et al. 2014b), where we analyze the relationship between MPD and the number of functions surpassing a threshold amount of functioning (e.g., number of plots surpassing $50 \%$ of the maximum function observed). 


\section{RESUlts}

\section{Simulations}

The three coexistence-based evolutionary processes created positive diversity-ecosystem function relationships (Fig. 1). For BM, ND, and OU niche evolution, ecosystem function increased with greater species richness and MPD (Fig. 1a-c), but the magnitude and strengths of these relationships depended on the model of evolution. BM produced strong correlations between species richness and ecosystem function, but generally weaker MPD-ecosystem function relationships. ND produced strong richness-ecosystem function and MPDecosystem function relationships. BM and ND models cannot be distinguished from richness-ecosystem function relationships, but do differ when examining MPDecosystem function relationships.

The OU model also tended to produce significant correlations between ecosystem function and both richness and MPD (Fig. 1c). However, these relationships were substantially weaker than those predicted by ND. More importantly, the relationships were flatter and OU models always yielded a lower maximum function.

Conversely, when clades evolve novel effect traits (NT), neither richness nor MPD was a significant predictor of ecosystem function (Fig. 1d). Furthermore, the signature of this type of mechanism is the higher variance among monocultures and reduced polyculture variance and a linear relationship with a slope $\approx 0$ (Fig. 1d).

Despite the fact that different evolutionary models were used to generate multiple ecosystem functions, the results of the multifunction analysis was remarkably similar to the single function results from BM niche evolution (Fig. S2). Both species richness and MPD were significant predictors of ecosystem function of average multifunction, but MPD tended to be relatively weak.

\section{Field experiment}

Of the ten ecosystem functions measured, five were significantly related to richness and MPD (average biomass, damage reduction, decomposition, structural complexity, and litter production - Figs. 2 and 3). Of these five functions, average biomass production (Figs. $2 \mathrm{~b}$ and $3 \mathrm{~b}$ ) was the most strongly related to both richness and MPD. Several other functions, especially litter production and structural complexity (Fig. 3a, c), were significantly related to MPD but with very low variance explained.

While it may be intuitive to dismiss the ecosystem functions that were not significantly explained by richness or MPD, -Delta N (Figs. $2 \mathrm{~h}$ and $3 \mathrm{~h}$ ) appeared to have a fixed mean value with relatively little variation around it, matching what could be expected under an OU model of evolution. Further, three other nonsignificant predictions (arthropod abundance, flower production, and pollinator abundance) appear to fit the data pattern expected under the evolution of novel effect traits. Indeed, two of these functions appear to be maximized in single clades (Fig. 4). Flower production was highest in the Solidago clade (Fig. 4b) and pollination visitation rates were highest in the Pycnanthemum clade (Fig. 4c). However, arthropod abundance, which appeared to fit the expected pattern from a novel clade (Fig. 3i), was not highest in a single clade (Fig. 4a), but instead several clades appeared to suppress arthropods (e.g., Pycnanthemum and the grass clades). Thus, the pattern expected by a clade of high functioning species (Fig. 1) could also be produced when a clade suppresses function.

MPD was a significant predictor of ecosystem multifunctionality, regardless of the multifunctionality measure employed (Fig. 5). However, the explanatory value of MPD is rather weak with substantial variability, and is similar to what is predicted by multiple traits evolving under Brownian motion evolution (Appendix S1: Fig. S1). Further, MPD was a better predictor at lower thresholds for polyculture function and had the best explanatory power at a threshold of just 11\% (Fig. 5).

\section{DISCUSSION}

The relationship between ecological differences and phylogenetic distances has been controversial (Narwani et al. 2013, Gerhold et al. 2015), yet most analyses do not use evolutionary models to generate ecological expectations (Mouquet et al. 2012, Cadotte et al. 2017), making it difficult to assess whether negative results reported in the literature are due to evolution not influencing ecological differences per se or that a specific (often unspecified) evolutionary model is not valid (Cadotte et al. 2017). Different models generate different relationships between phylogenetic distance and ecological differences, which influence the correlation between ecosystem function and both species richness and phylogenetic diversity. In our analysis, we use several models to generate expected diversity-ecosystem function relationships.

The evolution of traits and niche differences undoubtedly influence species' contributions to ecosystem function. However, the influence of species on function depends upon the model of evolution, as well as the way in which species' traits influence function (e.g., through their influence on coexistence vs. direct influence on function). Here we show that different models of evolution predict the strength of diversity-ecosystem function relationships. Further, combining multiple functions reveals the importance of diversity on ecosystem multifunctionality, but at the same time reduces the signatures of different models of evolution.

Using evolutionary models to generate expectations is important because the often-assumed relationship between phylogenetic diversity, species differences, and ecosystem function is simplistic. Previous analyses (e.g., Cadotte et al. 2008) assume that species differences are linearly related to phylogenetic differences, but this is not the precise prediction generated by a number of 
a) Litter

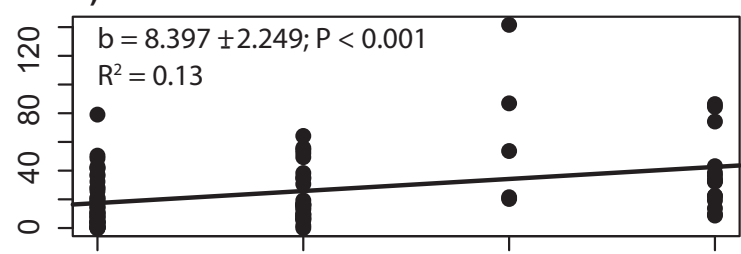

c) Structural complexity
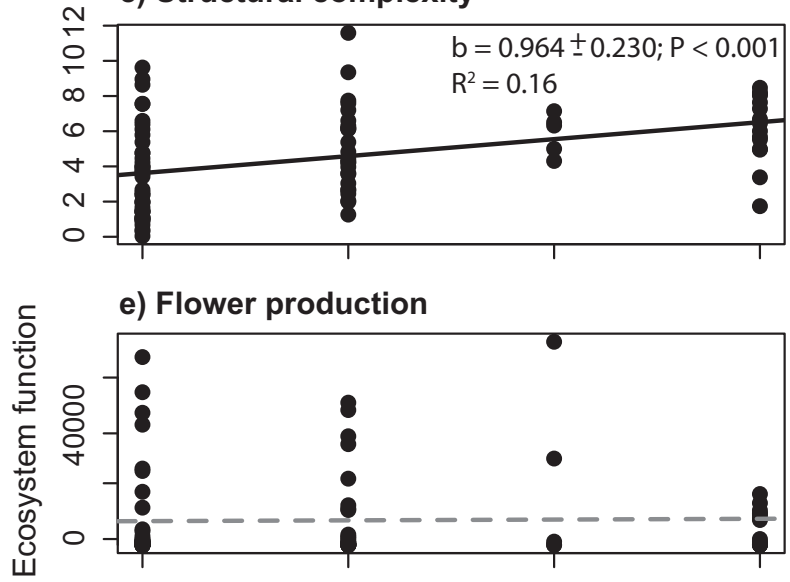

g) Damage reduction

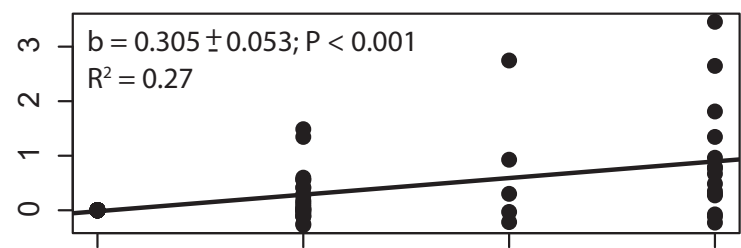

i) Arthropod abundance

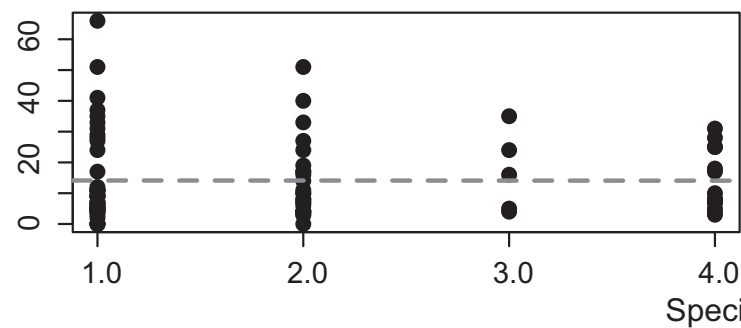

b) Average biomass

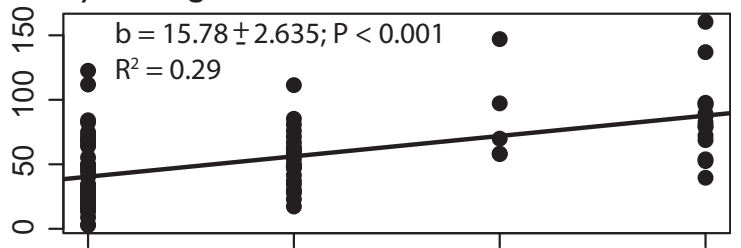

d) Pollinator abundance
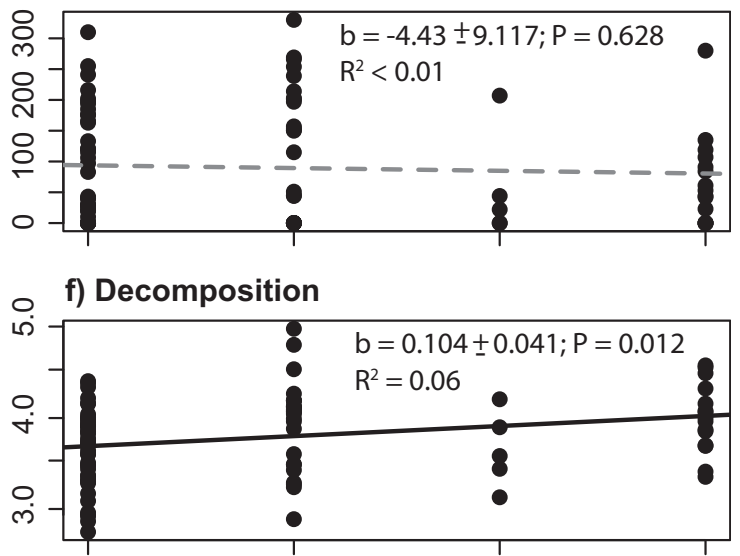

h) -Delta $N$

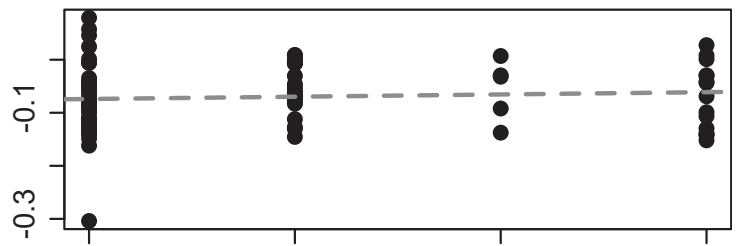

j) Arthropod richness

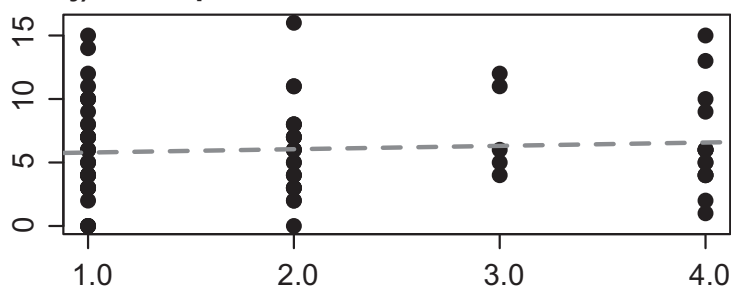

FIG. 2. The relationships between species richness and 10 different measures of ecosystem function. Solid lines indicate a significant relationship and dashed lines show non-significant ones.

evolutionary models (Cadotte et al. 2017). This distinction matters because debates about the relative value of species richness and phylogenetic diversity to explain ecosystem function (Cadotte 2015a, Cardinale et al. 2015, Venail et al. 2015) have been limited by a lack of evolutionary models. The question shouldn't be "is richness a better predictor of function than phylogenetic diversity", rather we should ask how different models of evolution influence diversity-ecosystem function relationships (Srivastava et al. 2012). In fact, non-significant diversity-ecosystem function relationships do not necessarily mean that evolutionary history does not influence function, since species contributions to function might be best described by an OU model or the evolution of novel traits that directly affect ecosystem function (e.g., Fig. 1).

There is mounting evidence that evolution can strongly influence ecosystem functions (but the reverse is likely true -that the ecological mechanisms that generate ecosystem function influence evolution, though this is not frequently tested). Our analyses reveal that several different models explain a number of different ecosystem functions. Productivity is strongly predicted by richness and MPD, as is decomposition rate and damage reduction, and here we conclude that these results appear consistent 
a) Litter

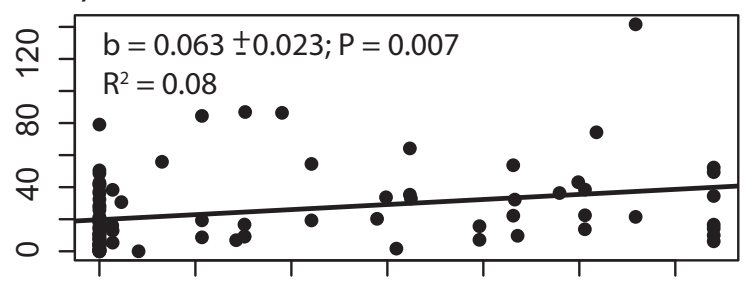

c) Structural complexity

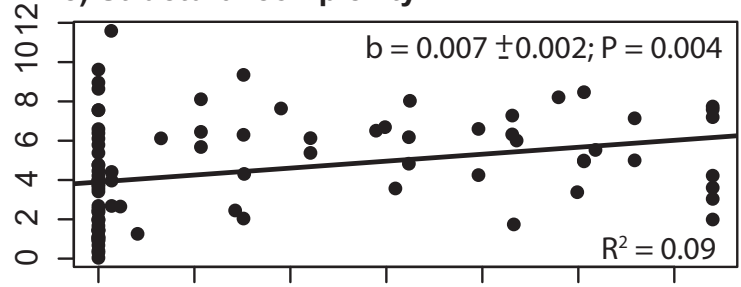

e) Flower production

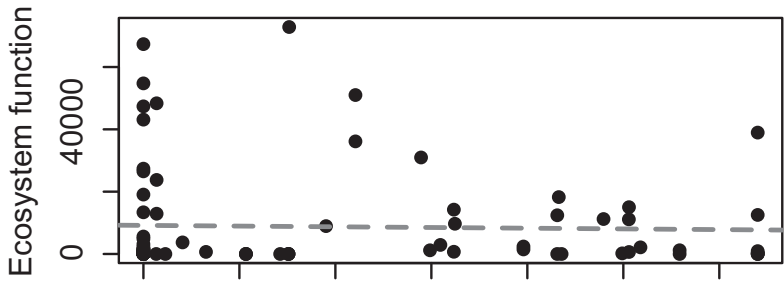

g) Damage reduction

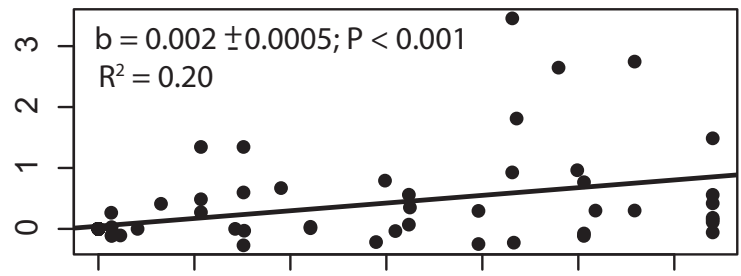

i) Arthropod abundance

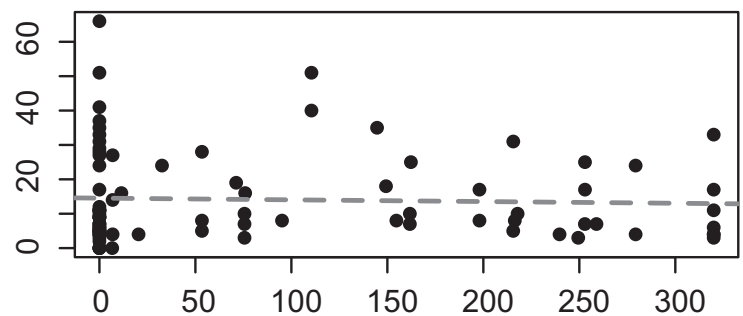

b) Average biomass

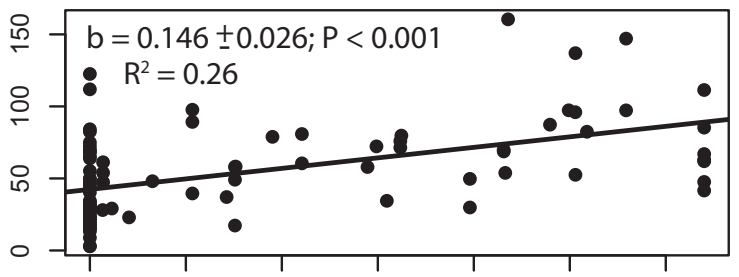

d) Pollinator abundance

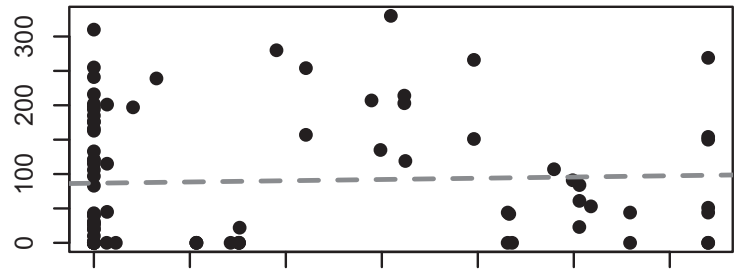

f) Decomposition

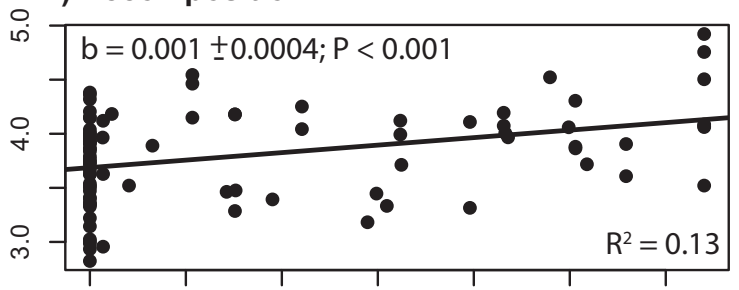

h) -Delta $N$

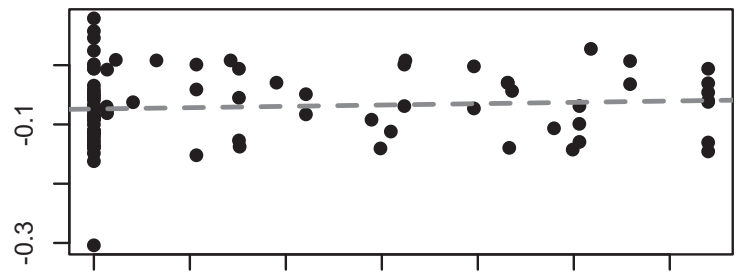

j) Arthropod richness

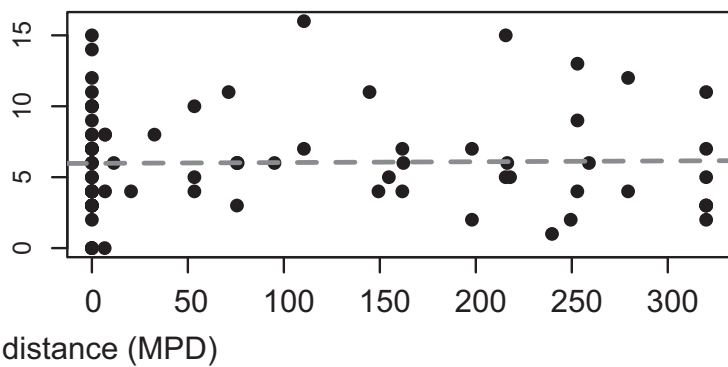

FIG. 3. The relationships between species mean pairwise distance (MPD) and 10 different measures of ecosystem function. Solid lines indicate a significant relationship and dashed lines show non-significant ones.

with evolution of trait differences from either Brownian motion or divergent niche evolution, but more sophisticated simulations and larger experiments are required. A couple of other functions (structural complexity and litter production) have a weaker relationship with MPD, but still appear consistent with Brownian motion evolution. A further three functions (Arthropod abundance, flower production, and pollinator abundance) are consistent with the evolution of traits within clades with direct effects on function, and produce non-significant diversity-ecosystem function relationships. Only two functions (arthropod richness and soil nitrogen depletion) were unrelated to richness and MPD and could be explained by an OU model of evolution, or just the fact that there is 
a)

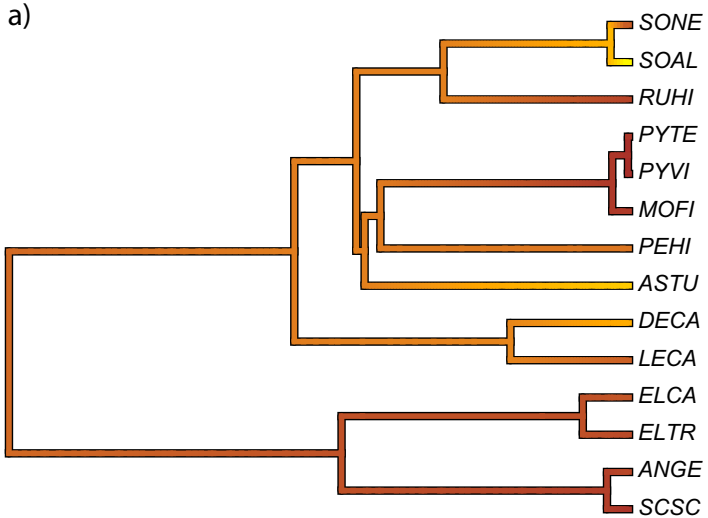

b)

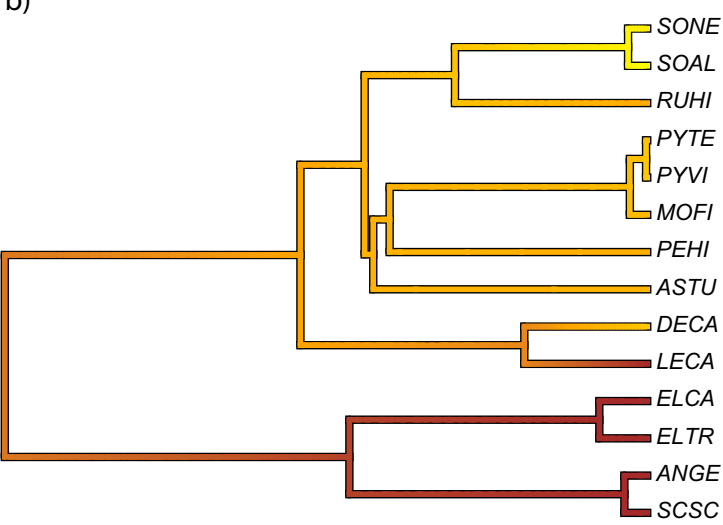

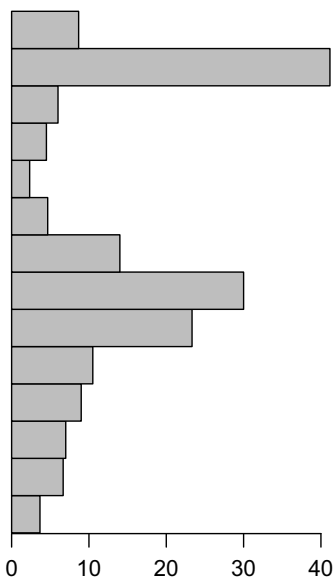

Average arthropod abundance

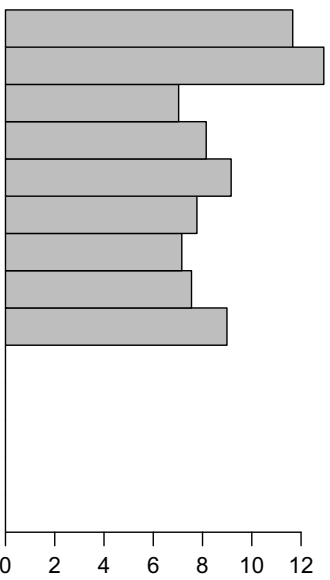

Log(cumulative number of flowers/biomass)

c)
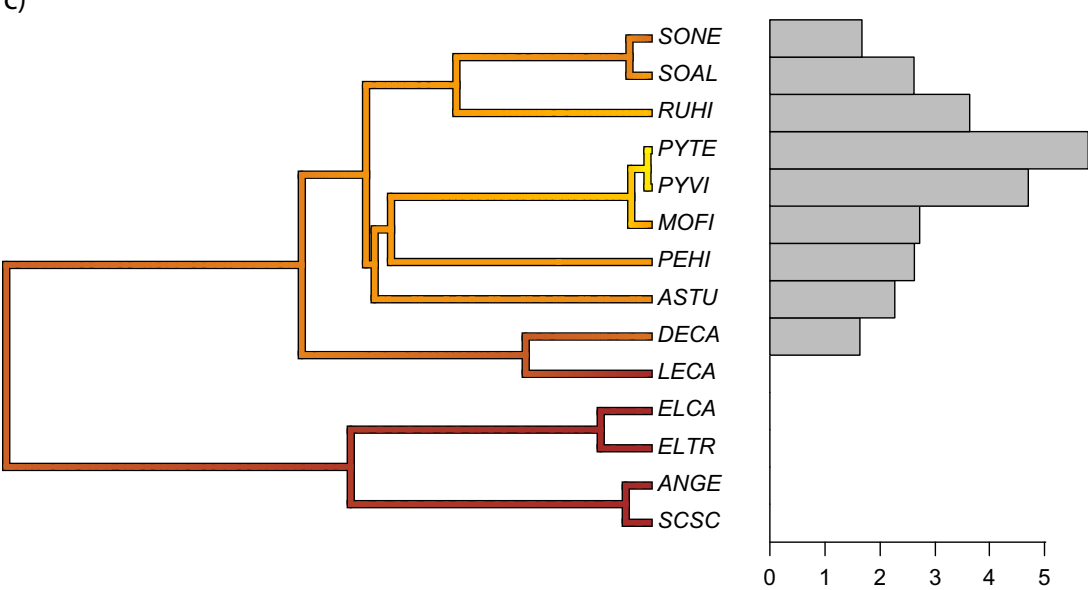

Log(cumulative pollinator visits/biomass)

FIG. 4. Species contributions to function across the phylogenetic tree for (a) arthropod abundance, (b) flower production; and (c) pollinator visitation. Species codes refer to: SONE: Solidago nemoralis; SOAL: S. altissima; RUHI: Rudbeckia hirta; PYTE: Pycnanthemum tenuifolium; PYVI: P. virginianum; MOFI: Monarda fistulosa; PEHI: Penstemon hirsutus; ASTU: Asclepias tuberosa; DECA: Desmodium canadense; LECA: Lespedeza capitata; ELCA: Elymus canadensis; ELTR: E. trachycaulu; ANGE: Andropogon gerardii; SCSC: Schizachyrium scoparium. 


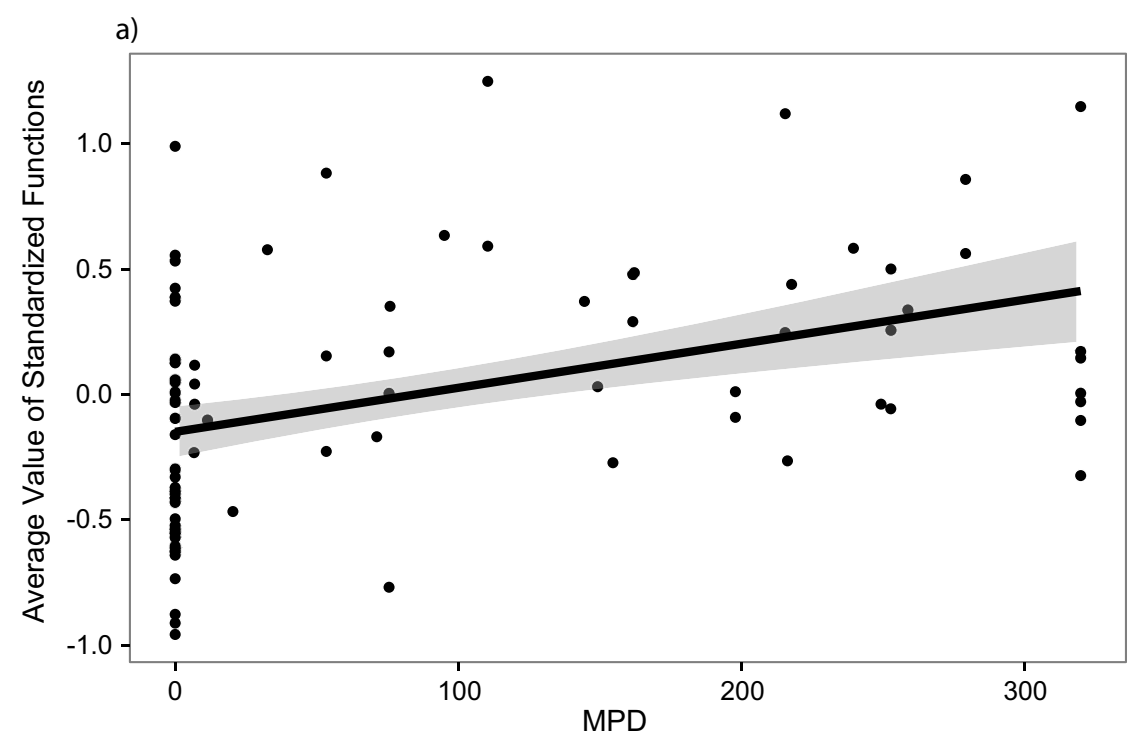

b)

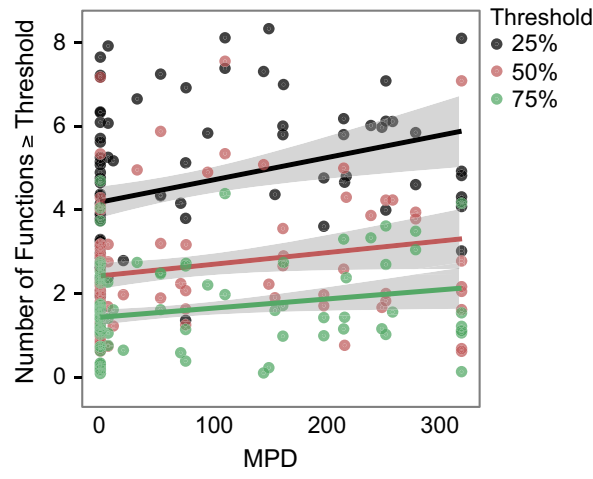

c)

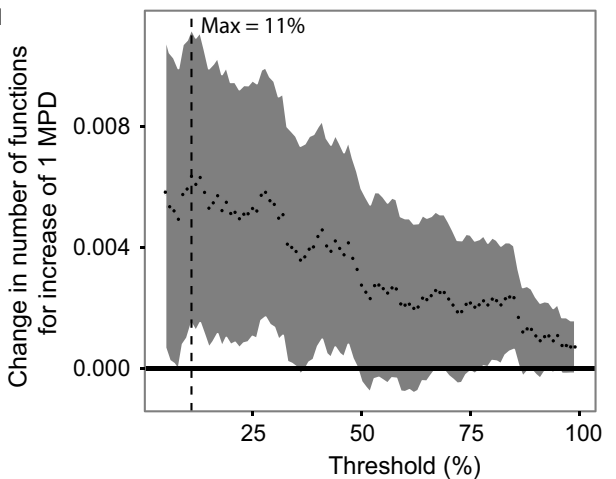

FIG. 5. Analysis of diversity-multifunctionality relationships. (a) The relationship between mean pairwise distance (MPD) and multifunction (measured as the standardize average function). (b) The relationship between MPD and the number of functions surpassing a threshold of functions, for three thresholds. (c) The strength of the relationship between MPD and the number of functions surpassing a threshold of functions, across a gradient of thresholds.

insufficient variation at the scale of our study. While we implicitly assume that species contributions to function are influenced by relatively simple models of evolution acting on relatively few traits, real patterns of evolution could be much more complex, including trait non-independence and traits evolving at different rates.

Several studies have shown how evolutionary history can influence individual ecosystem functions. Evolutionary diversification in stickleback fish has been shown to have important consequences for prey community structure and primary production when members of divergent lineages are combined (Harmon et al. 2009). Increased plant phylogenetic diversity is associated with greater soil microbial biomass and activity (Navarro-Cano et al. 2014), which could be responsible for the increased decomposition rates observed in the current study. Parker et al. (2015) found that decreasing plant phylogenetic diversity was associated with greater disease damage on rare species, revealing the phylogenetic non-independence of disease transfer in communities. Our results are compatible with this, and despite expectations that diseasepathogen interactions should rapidly evolve (Parker and Gilbert 2004), plant evolutionary history appears to be an important predictor of disease patterns.

The most important insights into how evolution can shape diversity-ecosystem function relationships come from microbial experiments (Gravel et al. 2011, 2012). In a series of experiments by Gravel et al. (2011, 2012), marine bacteria were subjected to differing selection regimes using artificial carbon substrates and the authors showed how evolution can alter the relationship between phylogenetic diversity and ecosystem function. Under selection regimes that create specialists, steeper diversity-ecosystem function relationships are observed, compared to evolved generalists (Gravel et al. 2011). Further, strong selection that increases average carbon use efficiency (similar to what is expected by OU evolution) can weaken these relationships (Gravel et al. 2012). 
While our results confirm that species' evolved differences contribute to ecosystem function, an additional factor that we did not consider is the role of intraspecific variability and plasticity in influencing ecosystem function (Burns and Strauss 2012, Violle et al. 2012, Wang et al. 2014). Work by Burns and Strauss (2011) has shown that closely related plant species are more likely to exhibit phenotypic plasticity, which contributes to their coexistence. Despite phylogenetically non-random plasticity, Burns and Strauss (2012), further showed that phylogeny still provided significant explanatory power for variation in biomass production. We could strengthen our understanding of ecosystem function by including plastic responses to competition in our analyses (Burns and Strauss 2012), however, we do not have the individual level data required to do this.

When we combined the 10 functions into a single multifunction measure, the MPD-multifunction relationship was significant, but was difficult to distinguish from that expected with a BM model of evolution (e.g., Appendix S1: Fig. S1). This result was consistent whether or not non-significant individual functions were excluded. While it is important to measure how ecosystem multifunctionality is influenced by assemblage diversity, it is also important to understand the relationships between diversity and individual functions if one wishes to understand mechanistic underpinnings (Byrnes et al. 2014a).

The most important limitation of this analysis is that our experiment was limited to a maximum diversity of just four species. Plant multifunction is likely maximized by much higher diversity (Zavaleta et al. 2010), and this is reinforced by the relatively weak threshold analyses (Fig. 5). Only about two functions per plot surpass a $75 \%$ threshold at four species (Fig. 5). Zavaleta et al. (2010) found that about 12 species were required to surpass a $60 \%$ threshold for four functions. Thus, many more species would be required to maximize 10 functions.

\section{Conclusions}

Given that human land use, habitat management, and restoration can influence species diversity, understanding how changes in diversity affect the provisioning of ecosystem functions and services is of utmost importance if we are to develop sound management solutions. Importantly, these functions and services can be manipulated in anthropogenically influenced landscapes by adopting practices that influence species diversity. But to do this, we need to understand why diversity influences function. Here we show that the evolutionary history of species can be modelled to make predictions about how phylogenetic diversity correlates with ecosystem function. In a field experiment, we show that different functions appear to be the product of different models of evolution, resulting in different types and strengths of diversity-function relationships. By understanding how species evolve we can predict species combinations that will maximize ecosystem function.

\section{ACKNOWLEDGMENTS}

MWC, JL and WS developed the questions and design of the study, and MWC analyzed the data and wrote the first draft of the manuscript. MWC, RD and RM designed and implemented the field experiment. MWC, SWL, SEY and JS collected data. All authors contributed to manuscript writing, revision and editing. This work was supported by the TD Professor of Urban Forest Conservation and Biology chair, Canada Foundation for Innovation, the Ontario Research Fund, and Natural Sciences and Engineering Research Council of Canada (no. 386151) to MWC. We wish to thank the follow people for field assistance: Mathura Anandarajah, Michelle Chin, Alice Choi, Melanie Elliot, Homa Javadzadeh, Shafak Joyia, Rhokini Kunanesan, Hussan Khun, Amy Lui, Nauman Malik, Shahin Moallem, Jake Munroe, Ryan Munroe, Ciprian Munteanu, Sivajanani Sivarajah, Sam Xing, Cindy Yang and Nai Zhang.

\section{Literature Cited}

Anisimova, M., and O. Gascuel. 2006. Approximate likelihood ratio test for branches: A fast accurate and powerful alternative. Systematic Biology 55:539-552.

Balvanera, P., A. B. Pfisterer, N. Buchmann, J.-S. He, T. Nakashizuka, D. Raffaelli, and B. Schmid. 2006. Quantifying the evidence for biodiversity effects on ecosystem functioning and services. Ecology Letters 9:1146-1156.

Beals, M. L. 2006. Understanding community structure: a datadriven multivariate approach. Oecologia 150:484-495.

Benson, D. A., I. Karsch-Mizrachi, D. J. Lipman, J. Ostell, and D. L. Wheeler. 2005. GenBank. Nucleic Acids Research 33: D34-D38.

Bradford, M. A., S. A. Wood, R. D. Bardgett, H. I. Black, M. Bonkowski, T. Eggers, S. J. Grayston, E. Kandeler, P. Manning, and H. Setälä. 2014. Discontinuity in the responses of ecosystem processes and multifunctionality to altered soil community composition. Proceedings of the National Academy of Sciences 111:14478-14483.

Burns, J. H., and S. Y. Strauss. 2011. More closely related species are more ecologically similar in an experimental test. Proceedings of the National Academy of Sciences 108:5302-5307.

Burns, J. H., and S. Y. Strauss. 2012. Effects of competition on phylogenetic signal and phenotypic plasticity in plant functional traits. Ecology 93:S126-S137.

Butler, M. A., and A. A. King. 2004. Phylogenetic comparative analysis: A modeling approach for adaptive evolution. American Naturalist 164:683-695.

Byrnes, J., J. S. Lefcheck, L. Gamfeldt, J. N. Griffin, F. Isbell, and A. Hector. 2014a. Multifunctionality does not imply that all functions are positively correlated. Proceedings of the National Academy of Sciences 111:201419515.

Byrnes, J. E., L. Gamfeldt, F. Isbell, J. S. Lefcheck, J. N. Griffin, A. Hector, B. J. Cardinale, D. U. Hooper, L. E. Dee, and J. Emmett Duffy. 2014b. Investigating the relationship between biodiversity and ecosystem multifunctionality: challenges and solutions. Methods in Ecology and Evolution $5: 111-124$

Cadotte, M. W. 2013. Experimental evidence that evolutionarily diverse assemblages result in higher productivity. Proceedings of the National Academy of Sciences 110:8996-9000.

Cadotte, M. W. 2015a. Phylogenetic diversity and productivity: gauging interpretations from experiments that do not manipulate phylogenetic diversity. Functional Ecology 29:16031606.

Cadotte, M. W. 2015b. Phylogenetic diversity-ecosystem function relationships are insensitive to phylogenetic edge lengths. Functional Ecology 29:718-723 in press. 
Cadotte, M. W., and T. J. Davies. 2016. Phylogenies in ecology: a guide to concepts and methods. Princeton University Press, Princeton, New Jersey, USA.

Cadotte, M. W., B. J. Cardinale, and T. H. Oakley. 2008. Evolutionary history and the effect of biodiversity on plant productivity. Proceedings of the National Academy of Sciences of the United States of America 105:17012-17017.

Cadotte, M. W., T. J. Davies, and P. R. Peres-Neto. 2017. Why phylogenies do not always predict ecological difference. Ecological Monographs. https://doi.org/10.1002/ecm.1267

Cardinale, B. J., D. S. Srivastava, J. E. Duffy, J. P. Wright, A. L. Downing, M. Sankaran, and C. Jouseau. 2006. Effects of biodiversity on the functioning of trophic groups and ecosystems. Nature 443:989-992.

Cardinale, B. J., et al. 2015. Further re-analyses looking for effects of phylogenetic diversity on community biomass and stability. Functional Ecology 29:1607-1610.

Carroll, I., B. Cardinale, and R. Nisbet. 2011. Niche and fitness differences relate the maintenance of diversity to ecosystem function. Ecology 92:1157-1165.

Chapin, F. S., et al. 2000. Consequences of changing biodiversity. Nature 405:234-242.

Clarke, M., G. H. Thomas, and R. P. Freckleton. 2015. Trait evolution in adaptive radiations: modelling and measuring interspecific competition on phylogenies. bioRxiv. https://doi. org/10.1101/033647

Costanza, R., et al. 1997. The value of the world's ecosystem services and natural capital. Nature 387:253-260.

Crutsinger, G. M., M. D. Collins, J. A. Fordyce, Z. Gompert, C. C. Nice, and N. J. Sanders. 2006. Plant genotypic diversity predicts community structure and governs an ecosystem process. Science 313:966-968.

Dooley, Á., F. Isbell, L. Kirwan, J. Connolly, J. A. Finn, and C. Brophy. 2015. Testing the effects of diversity on ecosystem multifunctionality using a multivariate model. Ecology Letters 18:1242-1251.

Edgar, R. C. 2004. MUSCLE: multiple sequence alignment with high accuracy and high throughput. Nucleic Acids Research 32:1792-1797.

Flynn, D. F. B., N. Mirotchnick, M. Jain, M. I. Palmer, and S. Naeem. 2011. Functional and phylogenetic diversity as predictors of biodiversity-ecosystem funciton relationships. Ecology 92:1573-1581.

Gamfeldt, L., H. Hillebrand, and P. R. Jonsson. 2008. Multiple functions increase the importance of biodiversity for overall ecosystem functioning. Ecology 89:1223-1231.

Gerhold, P., J. F. Cahill, M. Winter, I. V. Bartish, and A. Prinzing. 2015. Phylogenetic patterns are not proxies of community assembly mechanisms (they are far better). Functional Ecology 29:600-614.

Ghazoul, J. 2006. Floral diversity and the facilitation of pollination. Journal of Ecology 94:295-304.

Gravel, D., T. Bell, C. Barbera, T. Bouvier, T. Pommeir, P Venail, and N. Mouquet. 2011. Experimental niche evolution alters the strength of the diversity-productivity relationship. Nature 469:89-92.

Gravel, D., T. Bell, C. Barbera, M. Combe, T. Pommier, and N. Mouquet. 2012. Phylogenetic constraints on ecosystem functioning. Nature Communications 3:1117.

Guindon, S., and O. Gascuel. 2003. A simple, fast, and accurate algorithm to estimate large phylogenies by maximum likelihood. Systematic Biology 52:696-704.

Hansen, R., N. Frantzeskaki, T. McPhearson, E. Rall, N. Kabisch, A. Kaczorowska, J.-H. Kain, M. Artmann, and S. Pauleit. 2015. The uptake of the ecosystem services concept in planning discourses of European and American cities. Ecosystem Services 12:228-246.
Harmon, L. J., B. Matthews, S. Des Roches, J. M. Chase, J. B. Shurin, and D. Schluter. 2009. Evolutionary diversification in stickleback affects ecosystem functioning. Nature 458:11671170 .

Hector, A., and R. Bagchi. 2007. Biodiversity and ecosystem multifunctionality. Nature 448:188-190.

Klein, A. M., B. E. Vaissiere, J. H. Cane, I. Steffan-Dewenter, S. A. Cunningham, C. Kremen, and T. Tscharntke. 2007. Importance of pollinators in changing landscapes for world crops. Proceedings of the Royal Society B: Biological Sciences 274:303-313

Lavorel, S., K. Grigulis, P. Lamarque, M. P. Colace, D. Garden, J. Girel, G. Pellet, and R. Douzet. 2011. Using plant functional traits to understand the landscape distribution of multiple ecosystem services. Journal of Ecology 99:135-147.

Lefcheck, J. S., J. E. K. Byrnes, F. Isbell, L. Gamfeldt, J. N. Griffin, N. Eisenhauer, M. J. S. Hensel, A. Hector, B. J. Cardinale, and J. E. Duffy. 2015. Biodiversity enhances ecosystem multifunctionality across trophic levels and habitats. Nature Communications 6:6936.

Mace, G. M., K. Norris, and A. H. Fitter. 2012. Biodiversity and ecosystem services: a multilayered relationship. Trends in Ecology and Evolution 27:19-26.

Marshall, S. 2006. Insects: their natural history and diversity: with a photographic guide to insects of eastern North America. Firefly Books, Richmond Hill, Ontario, Canada.

Mouquet, N., et al. 2012. Ecophylogenetics: advances and perspectives. Biological Reviews 87:769-785.

Narwani, A., M. A. Alexandrou, T. H. Oakley, I. T. Carroll, and B. J. Cardinale. 2013. Experimental evidence that evolutionary relatedness does not affect the ecological mechanisms of coexistence in freshwater green algae. Ecology Letters 16:1373-1381.

Navarro-Cano, J. A., M. Goberna, A. Valiente-Banuet, A. Montesinos-Navarro, C. García, and M. Verdú. 2014. Plant phylodiversity enhances soil microbial productivity in facilitation-driven communities. Oecologia 174:909-920.

Nowak, D. J., R. E. Hoehn, A. R. Bodine, E. J. Greenfield, A. Ellis, T. A. Endreny, Y. Yang, T. Zhou, and R. Henry. 2013. Assessing urban forest effects and values: Toronto's urban forest.

Nuismer, S. L., and L. J. Harmon. 2015. Predicting rates of interspecific interaction from phylogenetic trees. Ecology Letters 18:17-27.

Paradis, E., J. Claude, and K. Strimmer. 2004. APE: analyses of phylogenetics and evolution in $\mathrm{R}$ language. Bioinformatics 20:289-290.

Parker, I. M., and G. S. Gilbert. 2004. The evolutionary ecology of novel plant-pathogen interactions. Annual Review of Ecology, Evolution, and Systematics 35:675-700.

Parker, I. M., M. Saunders, M. Bontrager, A. P. Weitz, R. Hendricks, R. Magarey, K. Suiter, and G. S. Gilbert. 2015. Phylogenetic structure and host abundance drive disease pressure in communities. Nature 520:542-544.

Posada, D., and K. A. Crandall. 1998. MODELTEST: testing the model of DNA substitution. Bioinformatics 14:817-818.

Posada, D., and K. A. Crandall. 2001. Selecting the best-fit model of nucleotide substitution. Systematic Biology 50:580-601.

R Development Core Team. 2013. R: A language and environment for statistical computing. R Foundation for Statistical Computing, Vienna, Austria.

Sanderson, M. J. 2002. Estimating absolute rates of molecular evolution and divergence times: a penalized likelihood approach. Molecular Biology and Evolution 19:101-109.

Scherer-Lorenzen, M., C. Palmborg, A. Prinz, and E. D. Schulze. 2003. The role of plant diversity and composition for nitrate leaching in grasslands. Ecology 84:1539-1559. 
Simpson, G. G. 1944. Tempo and mode in evolution. Columbia University Press, New York, New York, USA.

Spehn, E. M., et al. 2005. Ecosystem effects of biodiversity manipulations in European grasslands. Ecological Monographs 75:37-63.

Srivastava, D. S., M. W. Cadotte, A. A. M. MacDonald, N. Mirotchnick, and R. G. Marushia. 2012. Phylogenetic diversity and the functioning of ecosystems. Ecology Letters 15:637-648.

Tilman, D. 1999. The ecological consequences of changes in biodiversity: A search for general principles. Ecology 80:1455-1474.

Tilman, D., P. B. Reich, J. Knops, D. Wedin, T. Mielke, and C. Lehman. 2001. Diversity and productivity in a long-term grassland experiment. Science 294:843-845.

Turnbull, L. A., J. M. Levine, M. Loreau, and A. Hector. 2013. Coexistence, niches and biodiversity effects on ecosystem functioning. Ecology Letters 16:116-127.
Venail, P., et al. 2015. Species richness, but not phylogenetic diversity, influences community biomass production and temporal stability in a re-examination of 16 grassland biodiversity studies. Functional Ecology 29:615-626.

Violle, C., B. J. Enquist, B. J. McGill, L. Jiang, C. H. Albert, C. Hulshof, V. Jung, and J. Messier. 2012. The return of the variance: intraspecific variability in community ecology. Trends in Ecology and Evolution 27:244-252.

Wang, X.-Y., Y. Miao, S. Yu, X.-Y. Chen, and B. Schmid. 2014. Genotypic diversity of an invasive plant species promotes litter decomposition and associated processes. Oecologia 174:993-1005.

Zavaleta, E. S., J. R. Pasari, K. B. Hulvey, and G. D. Tilman. 2010. Sustaining multiple ecosystem functions in grassland communities requires higher biodiversity. Proceedings of the National Academy of Sciences of the United States of America 107:1443-1446.

\section{SUPPORTING INFORMATION}

Additional supporting information may be found in the online version of this article at http://onlinelibrary.wiley.com/doi/ 10.1002/ecy.2045/suppinfo 